\title{
Response to: Risk Factors for Cement Loosening after Vertebroplasty for Osteoporotic Vertebral Fracture with Intravertebral Cleft: A Retrospective Analysis
}

\author{
Toshio Nakamae ${ }^{1}$, Kiyotaka Yamada ${ }^{1}$, Yasuyuki Tsuchida ${ }^{2}$, \\ Orso Lorenzo Osti ${ }^{3}$, Nobuo Adachi ${ }^{4}$, Yoshinori Fujimoto ${ }^{1}$ \\ ${ }^{1}$ Department of Orthopaedic Surgery, JA Hiroshima General Hospital, Hiroshima, Japan \\ ${ }^{2}$ Department of Radiology, JA Hiroshima General Hospital, Hiroshima, Japan \\ ${ }^{3}$ Spinal Service, Calvary Health Care, North Adelaide Campus, North Adelaide, SA, Australia \\ ${ }^{4}$ Department of Orthopaedic Surgery, Integrated Health Sciences, Institute of Biomedical and Health Sciences, Hiroshima University, Hiroshima, Japan
}

We fully appreciate the letter regarding our manuscript entitled "Risk factors for cement loosening after vertebroplasty for osteoporotic vertebral fracture with intravertebral cleft: a retrospective analysis [1].” We thank you who carefully read our manuscript and provided valuable comments.

We do agree that there is clearly a dearth of an accepted classification system for osteoporotic vertebral fracture management and it is reasonable to amalgamate osteoporotic vertebral fracture classification and thoracolumbar traumatic fracture classifications in a non-osteoporotic patient. However, we should keep in mind that osteoporotic vertebral fracture is different from the traumatic fracture in young patients in terms of the fracture pathophysiology.

We identified the unstable vertebral fracture (spinous process fracture (posterior column injury), marked intravertebral instability and split fracture) lead to cement loosening after percutaneous vertebroplasty (PVP) in osteoporotic vertebral fracture with intravertebral cleft.
In those patients, we should observe carefully after PVP and sometimes we need instrumented surgery. It is urgent business for us to establish the osteoporotic vertebral fracture classification in terms of the stability.

Although you mentioned that PVP is not ideal indication for the patients associated with neurological deficit, we reported that PVP is one of the treatment options for the osteoporotic vertebral fracture with neurological deficit when we choose the patient appropriately [2]. We sincerely thank you again for your interest in our article.

\section{Conflict of Interest}

No potential conflict of interest relevant to this article was reported.

\section{References}

1. Nakamae T, Yamada K, Tsuchida Y, Osti OL, Adachi N, Fujimoto Y. Risk factors for cement loosening

Received Dec 5, 2018; Accepted Dec 10, 2018

Corresponding author: Toshio Nakamae

Department of Orthopaedic Surgery, JA Hiroshima General Hospital, Hiroshima, Japan, 1-3-3 Jigozen, Hatsukaichi, Hiroshima, 7388503, Japan

Tel: +81-829-36-3111, Fax: +81-829-36-5573, E-mail: toshinakamae623813@yahoo.co.jp 
after vertebroplasty for osteoporotic vertebral fracture with intravertebral cleft: a retrospective analysis. Asian Spine J 2018;12:935-42.

2. Nakamae T, Fujimoto Y, Yamada K, Takata H, Shim- bo T, Tsuchida Y. Percutaneous vertebroplasty for osteoporotic vertebral compression fracture with intravertebral cleft associated with delayed neurologic deficit. Eur Spine J 2013;22:1624-32. 